\title{
La transculturación de una familia de migrantes mixtecos de la colonia Ferrocarril a través de la comunicación cultural: Estudio de caso
}

\author{
María del Carmen Padilla Arreguín* \\ Asesor de Tesis: Verónica Victorica Pérez \\ Universidad del VAlle de Atemajac, Jalisco
}

Esta investigación surge por el interés en los múltiples cambios culturales que se dan dentro de la metrópoli; la compleja convivencia de diferentes grupos; la interculturalidad particular de la que forman parte los grupos indígenas que migran a las ciudades; así como por el movimiento social y los cambios que ocurren en las familias migrantes y en los habitantes del lugar de recepción.

Este trabajo es un estudio de caso enfocado en una familia de migrantes mixtecos de la colonia Ferrocarril, sumergida en la urbe, en la zona metropolitana de Guadalajara.

Es importante mencionar que el proceso de cambio cultural es complejo y ocurre como resultado de una aceptación o rechazo por parte del grupo migrante hacia la cultura urbana, en donde intervienen varios factores en los que la familia se encuentra en permanente interacción comunicativa, como son: la escuela, el trabajo, la religión, los medios de comunicación y las relaciones interpersonales, entre otros.

El estudio se orientó a describir la transculturación de una familia de migrantes mixtecos de la colonia Ferrocarril, con un enfoque de comunicación intercultural. Para ello, se establecieron las siguientes categorías como ejes del cambio cultural, las cuales están interrelacionadas con procesos de comunicación y cultura: festividades religiosas, red de relaciones, lengua, artesanías e indumentaria .

* Correo electrónico: carmenpadillaarreguin@hotmail.com 
El contexto social forma parte importante del trabajo, de manera que los sucesos que anteceden, y los procesos sociales que lo contextualizan, enriquecen la investigación, logrando un mayor argumento.

Esta investigación se basó en tres tópicos centrales: indigenismo, cultura y migración, los cuales le dan mayor amplitud y comprensión al estudio.

Por otra parte, las teorías que fundamentan el trabajo teórico, de campo y el análisis, tienen tres vertientes: la primera es la teoría de la "hermenéutica profunda", de John B. Thompson, que permite, a través de su marco metodológico, la consecución de tres análisis: el histórico, el discursivo y el interpretativo. La segunda teoría es de Néstor García Canclini, llamada "Hacia una teoría social del patrimonio", la cual ayuda a comprender, desde otro enfoque, la formación y el desarrollo del patrimonio cultural. Por último, y no menos importante, se plantea "la comunicación intercultural”, basada en varios autores, la cual enriquece aún más los fundamentos teóricos y le da una orientación distintiva al estudio. Los tres planteamientos teóricos exponen una interrelación, aunque conducidos de diferente manera, pero coinciden con los ejes centrales: los sujetos activos, su desarrollo y la comunicación de su cultura. Asimismo, las tres corrientes teóricas mantienen una continuidad en el desarrollo del trabajo, culminando con el análisis y las reflexiones finales.

En el cuadro 1 se presenta una relación de los tres enfoques teóricos en el estudio, su aplicación en el trabajo de campo y en el análisis.

Cuadro 1.

Relación entre los tres enfoques teóricos de la investigación

\begin{tabular}{|c|c|c|c|c|c|c|}
\hline & $\begin{array}{l}\text { TEORÍAS O } \\
\text { ENFOQUES }\end{array}$ & AUTORES & $\begin{array}{l}\text { MARCO TEÓRICO } \\
\text { Y REFERENCIAL }\end{array}$ & $\begin{array}{c}\text { TRABAJO DE } \\
\text { CAMPO }\end{array}$ & \multirow{4}{*}{ 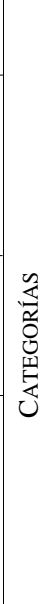 } & ANÁLISIS \\
\hline 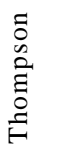 & $\begin{array}{c}\text { Teoría } \\
\text { metodológica: } \\
\text { la hermenéutica } \\
\text { profunda }\end{array}$ & $\begin{array}{l}\text { John B. } \\
\text { Thompson }\end{array}$ & $\begin{array}{c}\text { Análisis } \\
\text { sociohistórico }\end{array}$ & $\begin{array}{c}\text { Análisis } \\
\text { discursivo }\end{array}$ & & $\begin{array}{c}\text { Análisis } \\
\text { interpretativo }\end{array}$ \\
\hline 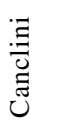 & $\begin{array}{l}\text { Hacia una } \\
\text { teoría social del } \\
\text { patrimonio }\end{array}$ & $\begin{array}{c}\text { Néstor García } \\
\text { Canclini }\end{array}$ & $\begin{array}{c}\text { Interacción de } \\
\text { la cultura }\end{array}$ & Interacción & & $\begin{array}{l}\text { Enfoque } \\
\text { cultural }\end{array}$ \\
\hline 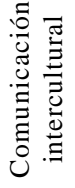 & $\begin{array}{l}\text { Comunicación } \\
\text { intercultural }\end{array}$ & $\begin{array}{c}\text { William B. } \\
\text { Gudykunst } \\
\text { y Miguel } \\
\text { Rodrigo Alsina }\end{array}$ & $\begin{array}{c}\text { Relación entre } \\
\text { comunicación } \\
\text { y cultura }\end{array}$ & $\begin{array}{c}\text { Procesos de } \\
\text { comunicación }\end{array}$ & & $\begin{array}{c}\text { Enfoque } \\
\text { comunicativo }\end{array}$ \\
\hline
\end{tabular}


La presente investigación se llevó a cabo a través de la metodología cualitativa. El método que se aplicó fue la etnografía, ya que se trata de un acercamiento donde se busca presentar la vivencia con la familia indígena. Cabe mencionar que la metodología cualitativa tiene relación con los enfoques de las teorías aplicadas, por lo que a las categorías en cuestión se les realiza un análisis de descripción, interpretación y relación de cultura-comunicación.

Por otra parte, las técnicas que se utilizaron fueron: la observación directa, participante y una entrevista semiestructurada, con el objetivo de tener un primer acercamiento a la realidad. Partiendo de estas técnicas se encontraron las categorías a desarrollar para la descripción y análisis del trabajo, las cuales son la base del análisis. Asimismo, las categorías tienen un sentido y un significado para la comunidad, y poseen un valor específico, que lo manifiestan en sus expresiones.

En esta investigación, los procesos comunicativos que se interrelacionaron con las categorías encontradas fueron: comunicación interpersonal, intergrupal, empatía, comunicación verbal y no verbal, competencia comunicativa e identidad, los cuales son un medio para que se lleve a cabo la transculturación de la familia de mixtecos de la colonia ferrocarril (ver Cuadro 2).

Es importante reflexionar sobre la modificación inevitable de los grupos de la ciudad, como parte de la comunicación intercultural que surge en ella, sobre la visión que tiene una familia indígena acerca de la transformación de su cultura, y de la importancia de la comunicación interpersonal entre los mixtecos de la colonia Ferrocarril, para rescatar una identidad propia y, junto con ello, lograr una mejor calidad de vida para los indígenas que viven en la ciudad.

El que integren patrones culturales ajenos y mantengan patrones de origen no significa que muera la cultura, sólo se transforma; y es que todo elemento en vida está en constante cambio, y todo sujeto en vida es parte de él. El que integren elementos culturales o se adapten a ellos habla de una capacidad comunicativa de los mixtecos para hacer de la familia o el grupo una cultura propia.

Por último, se debe recalcar que los cambios culturales son parte del desarrollo de las sociedades, que las culturas permanecen en constante transformación, y que la interacción comunicativa entre diferentes grupos culturales es vital para formar una sociedad pluricultural que, definitivamente, es dinámica y cambiante. 
Cuadro 2.

Resultados encontrados a través de las categorías de análisis entre los procesos de comunicación su interrelación e interpretación

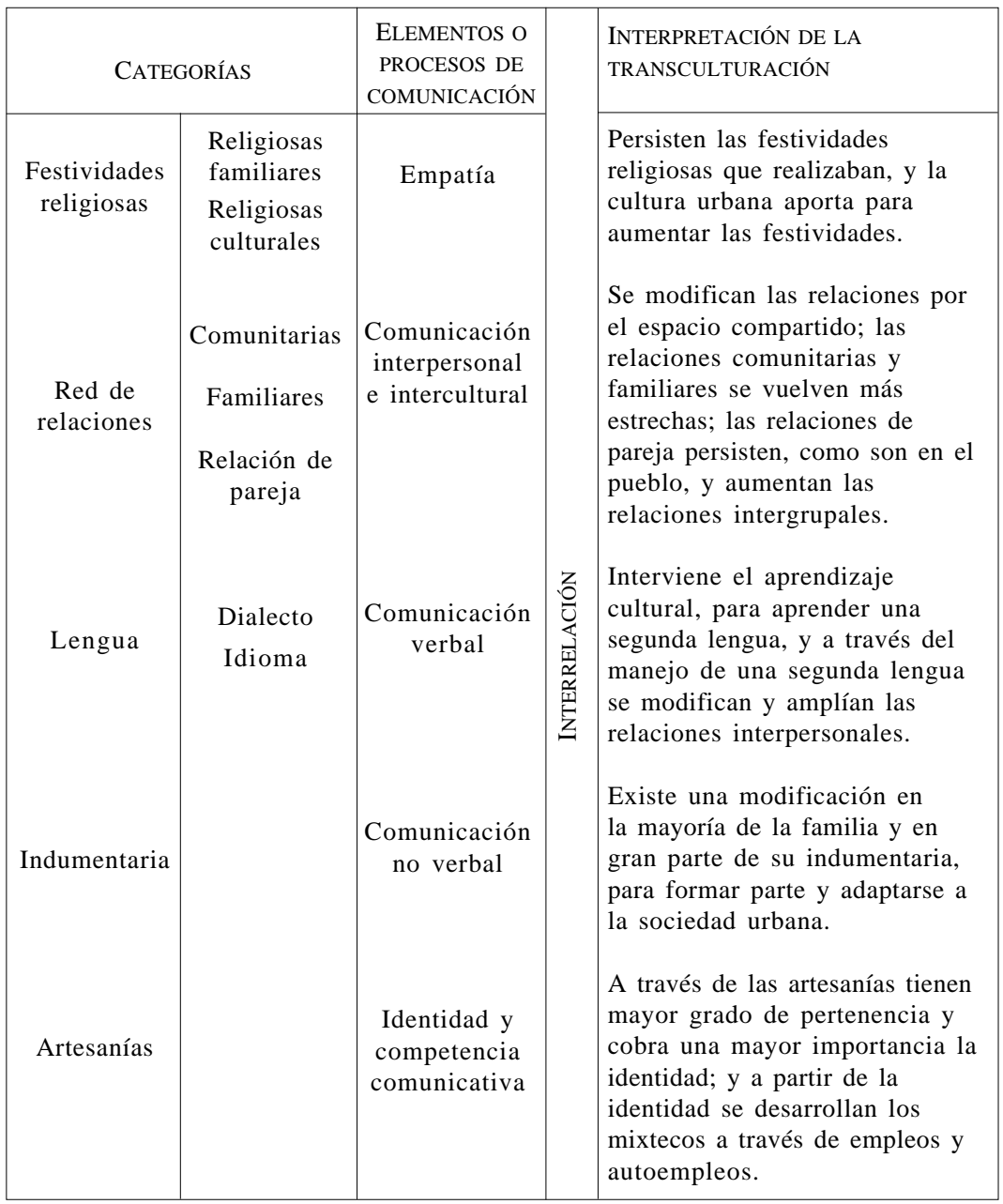

\section{BIBLIOGRAFÍA}

García Canclini, N. (1990) Culturas híbridas. México, D.F.: Grijalbo.

Gudykunst, William B. (2002) International and intercultural comunication (2a. ed.) Estados Unidos: Sage Publications.

Rodrigo Alsina, Miguel. (1999). La comunicación intercultural (1a. ed.).

Barcelona: Anthropos. 
Thompson, J. (1998). Ideología y cultura moderna. México: Universidad Autónoma Metropolitana.

Taylor, S.J. \& Bodgan, R. (1994). Introducción a los métodos cualitativos de la investigación. Barcelona: Paidós. 